PHYSICAL REVIEW D 96, 116014 (2017)

\title{
Addendum: New approach to the resummation of logarithms in Higgs-boson decays to a vector quarkonium plus a photon [Phys. Rev. D 95, 054018 (2017)]
}

\author{
Geoffrey T. Bodwin, ${ }^{1, *}$ Hee Sok Chung, ${ }^{2, \dagger}$ June-Haak Ee, ${ }^{3, *}$ and Jungil Lee ${ }^{3, \S}$ \\ ${ }^{1}$ High Energy Physics Division, Argonne National Laboratory, Argonne, Illinois 60439, USA \\ ${ }^{2}$ Theory Department, CERN, 1211 Geneva 23, Switzerland \\ ${ }^{3}$ Department of Physics, Korea University, Seoul 02841, Korea
}

(Received 7 October 2017; published 20 December 2017)

\begin{abstract}
In this addendum to Phys. Rev. D 95, 054018 (2017) we recompute the rates for the decays of the Higgs boson to a vector quarkonium plus a photon, where the vector quarkonium is $J / \psi, \Upsilon(1 S), \Upsilon(2 S)$, or $\Upsilon(3 S)$. We correct an error in the Abel-Padé summation formula that was used to carry out the evolution of the quarkonium light-cone distribution amplitude in Phys. Rev. D 95, 054018 (2017). We also correct an error in the scale of quarkonium wave function at the origin in Phys. Rev. D 95, 054018 (2017) and introduce several additional refinements in the calculation.
\end{abstract}

DOI: 10.1103/PhysRevD.96.116014

In Ref. [1], rates for the decays of the Higgs boson to a vector heavy quarkonium plus a photon $(H \rightarrow V+\gamma)$ were computed by making use of the light-cone amplitude for the direct decay process. In the direct process, the Higgs boson decays into a heavy-quark-antiquark pair $(Q \bar{Q})$, which radiates a gluon and evolves into a vector heavy quarkonium. In this addendum to Ref. [1], we make several improvements to the calculation of the direct amplitude in Ref. [1] and also correct an error in the scale of the heavyquarkonium wave function at the origin and an error in the application of the Abel-Padé method to the evolution of the light-cone distribution amplitude (LCDA).

In Ref. [1], $\phi_{V}(x)$, the heavy-quarkonium light-cone distribution amplitude at light-cone momentum fraction $x$, was evolved from the initial scale $\mu_{0}$, which is of order the heavy-quark mass, to the final scale $\mu$, which is of order the Higgs-boson mass $m_{H}$, by making use of the Abel-Padé method.

As was explained in Ref. [1], the Abel summation in the Abel-Padé method defines generalized functions (distributions) in the nonrelativistic QCD (NRQCD) expansion of the LCDA at the initial scale $\mu_{0}$ as limits of sequences of ordinary functions. In Eq. (31) of Ref. [1], the Abel summation was erroneously applied to $\phi_{V}(\mu)$, rather than to $\phi_{V}\left(\mu_{0}\right)$. Equation (31) of Ref. [1] should be corrected to the following:

\footnotetext{
*gtb@anl.gov

hee.sok.chung@cern.ch

*chodigi@gmail.com

§ungil@korea.ac.kr
}

Published by the American Physical Society under the terms of the Creative Commons Attribution 4.0 International license. Further distribution of this work must maintain attribution to the author(s) and the published article's title, journal citation, and DOI.

$$
\mathcal{M}=\lim _{z \rightarrow 1} \sum_{n, m=0}^{\infty} T_{m}(\mu) U_{m n}\left(\mu, \mu_{0}\right) z^{n} \phi_{n}^{\perp}\left(\mu_{0}\right)
$$

Here, $\mathcal{M}$ is the light-cone amplitude, $\phi_{n}^{\perp}\left(\mu_{0}\right)$ is the $n$th Gegenbauer moment of $\phi_{V}^{\perp}\left(x, \mu_{0}\right)$ [Eq. (19b) of Ref. [1]], $U_{m n}\left(\mu, \mu_{0}\right)$ is the evolution matrix for the LCDA [Eq. (B1) of Ref. [1]], and $T_{m}$ is the $m$ th Gegenbauer moment of the hard-scattering amplitude [Eq. (20b) of Ref. [1]]. The essential change in Eq. (1) is to replace $z^{m}$ with $z^{n}$.

Because the evolution matrix in leading logarithmic (LL) order is diagonal, this change affects the expression for the light-cone amplitude only at next-to-leading logarithmic (NLL) order and higher. In Ref. [1], only the LCDA at leading order in $\alpha_{s}$ and $v$ was evolved at NLL order. (Here, $\alpha_{s}$ is the QCD running coupling and $v$ is the velocity of the $Q$ or $\bar{Q}$ in the quarkonium rest frame.) Because the sums in Eq. (1) are not absolutely convergent, it is not obvious that the correction in Eq. (1) would not affect the results. However, it turns out that the correction in Eq. (1) does not shift the numerical results for the direct amplitudes significantly from those that were reported in Ref. [1], although it does improve the stability of the convergence of the Abel-Padé procedure.

The light-cone amplitude for the direct process can be written [2], by virtue of NRQCD factorization [3], as a sum of products of short-distance coefficients with NRQCD long-distance matrix elements (LDMEs) [4]. We denote the NRQCD LDME of leading order in $v$ by $\left\langle\mathcal{O}_{1}^{V}\right\rangle$. The quantity $\left\langle\mathcal{O}_{1}^{V}\right\rangle$ can be written in terms of the quarkonium wave function at the origin $\Psi_{V}(0)$ (Ref. [3]):

$$
\left\langle\mathcal{O}_{1}^{V}\right\rangle=\frac{1}{2 N_{c}}\left|\Psi_{V}(0)\right|^{2},
$$

where $N_{c}=C_{A}=3$ for $\mathrm{SU}(3)$ color. 
For the computations in Ref. [1], which involve $J / \psi$ and $\Upsilon(n S)$ final states, with $n=1,2$, or 3 , values for $\Psi_{V}(0)$ were inferred from values of $\left\langle\mathcal{O}_{1}^{V}\right\rangle$ that were given in Refs. [5,6]. In those papers, $\left\langle\mathcal{O}_{1}^{V}\right\rangle$ was computed by making use of the NRQCD expansion of the electronic decay rate $\Gamma\left(V \rightarrow e^{+} e^{-}\right)$through orders $\alpha_{s}(\mu)$ and $v^{2} .^{1}$ That expansion can be obtained from Eq. (2), the relation

$$
\Gamma\left(V \rightarrow e^{+} e^{-}\right)=\frac{4 \pi}{3 m_{V}} \alpha^{2}\left(m_{V}\right) e_{Q}^{2} f_{V}^{\| 2},
$$

and the NRQCD expansion

$$
\begin{aligned}
f_{V}^{\|}= & \frac{\sqrt{2 N_{c}} \sqrt{2 m_{V}}}{m_{V}} \Psi_{V}(0) \\
& \times\left[1-\frac{1}{6}\left\langle v^{2}\right\rangle_{V}-8 \frac{\alpha_{s}(\mu) C_{F}}{4 \pi}+O\left(\alpha_{s}^{2}, \alpha_{s} v^{2}, v^{4}\right)\right] .
\end{aligned}
$$

Here, $f_{V}^{\|}$is the decay constant for a longitudinally polarized quarkonium, $\alpha(\mu)$ is the electromagnetic fine-structure constant at the scale $\mu, e_{Q}$ is the heavy-quark charge, $m_{V}$ is the quarkonium mass, $C_{F}=\left(N_{c}^{2}-1\right) /\left(2 N_{c}\right)$, and $\left\langle v^{2}\right\rangle_{V}$ is the ratio of the NRQCD decay LDME of relative order $v^{2}$ to the NRQCD decay LDME of leading order in $v$ $\left(\left\langle\mathcal{O}_{1}^{V}\right\rangle\right)$. (See Eq. (6) of Ref. [1].) In Refs. [5,6], the scale $\mu$ in Eq. (4) was taken to be $m_{V}$. Hence, the values of $\left\langle\mathcal{O}_{1}^{V}\right\rangle_{V}$ that are given in those papers are at the scale $m_{V}$.

The decay rate for the process $H \rightarrow V+\gamma$ depends on the decay constant for a transversely polarized quarkonium $f_{V}^{\perp}(\mu)$, which has the NRQCD expansion $[4,7]$

$$
\begin{aligned}
f_{V}^{\perp}(\mu)= & \frac{\sqrt{2 N_{c}} \sqrt{2 m_{V}}}{2 m_{Q}} \Psi_{V}(0)\left[1-\frac{5}{6}\left\langle v^{2}\right\rangle_{V}\right. \\
& \left.+\left(-\log \frac{\mu^{2}}{m_{Q}^{2}}-8\right) \frac{\alpha_{s}(\mu) C_{F}}{4 \pi}+O\left(\alpha_{s}^{2}, \alpha_{s} v^{2}, v^{4}\right)\right],
\end{aligned}
$$

where $m_{Q}$ is the heavy-quark pole mass.

In Ref. [1], $f_{V}^{\perp}(\mu)$ was assumed to be at the scale $\mu=m_{Q}$. However, the values of $\Psi_{V}(0)$ that were used in Ref. [1] were inferred from the values of $\left\langle\mathcal{O}_{1}^{V}\right\rangle$ in
Refs. [5,6]. Because those values of $\left\langle\mathcal{O}_{1}^{V}\right\rangle$ are at the scale $\mu=m_{V}$, it is necessary to correct for the difference in scales. One can do this straightforwardly by making the following replacement in the expression for $f_{V}^{\perp}$ in Eq. (5):

$$
\Psi_{V}(0) \rightarrow \frac{1-\frac{1}{6}\left\langle v^{2}\right\rangle_{V}-8 \frac{C_{F} \alpha_{s}\left(m_{V}\right)}{4 \pi}}{1-\frac{1}{6}\left\langle v^{2}\right\rangle_{V}-8 \frac{C_{F} \alpha_{s}\left(m_{Q}\right)}{4 \pi}} \Psi_{V}(0) .
$$

Instead, we follow a procedure that was suggested in Ref. [8]. We compute $f_{V}^{\|}$from the measured quarkonium electronic decay rates, using Eq. (3). Then, we use Eq. (4) to eliminate $\Psi_{V}(0)$ from the amplitude and use Eqs. (4) and (5) to express $f_{V}^{\perp}$ in terms of $f_{V}^{\|}$:

$$
\begin{aligned}
f_{V}^{\perp}(\mu)= & \frac{m_{V}}{2 m_{Q}}\left[1-\frac{2}{3}\left\langle v^{2}\right\rangle_{V}-\frac{\alpha_{s}(\mu) C_{F}}{4 \pi} \log \frac{\mu^{2}}{m_{Q}^{2}}\right. \\
& \left.+O\left(\alpha_{s}^{2}, \alpha_{s} v^{2}, v^{4}\right)\right] f_{V}^{\|} \\
= & \frac{m_{V}}{2 \bar{m}_{Q}}\left[1-\frac{2}{3}\left\langle v^{2}\right\rangle_{V}+\frac{\alpha_{s}(\mu) C_{F}}{4 \pi}\left(-\log \frac{\mu^{2}}{\bar{m}_{Q}^{2}}-4\right)\right. \\
& \left.+O\left(\alpha_{s}^{2}, \alpha_{s} v^{2}, v^{4}\right)\right] f_{V}^{\|} .
\end{aligned}
$$

Because the pole mass is subject to renormalon ambiguities and, hence, is ill defined, we have used the one-loop relation between the $\overline{\mathrm{MS}}$ mass $\bar{m}_{Q}$ at the scale $\bar{m}_{Q}$ and the pole mass [9], namely,

$$
m_{Q}=\bar{m}_{Q}\left[1+C_{F} \frac{\alpha_{s}\left(\bar{m}_{Q}\right)}{\pi}+O\left(\alpha_{s}^{2}\right)\right],
$$

to express $f_{V}^{\perp} / f_{V}^{\|}$in terms of the $\overline{\mathrm{MS}}$ mass in Eq. (7b). As can be seen, if $\mu$ is approximately equal either to $m_{Q}$ or to $\bar{m}_{Q}$, then the apparent rates of convergence of both the velocity expansions and the $\alpha_{s}$ expansions in Eqs. (7a) and (7b) are better than the corresponding rates of convergence in Eq. (5). Furthermore, the removal of $\Psi_{V}(0)$ from the amplitude reduces some of the theoretical uncertainties. The resulting expression for the direct amplitude, which corresponds to Eq. (37) in Ref. [1], is

$$
\begin{aligned}
i \mathcal{M}_{\mathrm{dir}}^{\mathrm{LC}}[H \rightarrow V+\gamma]= & \frac{i}{2} e e_{Q} \kappa_{Q} \bar{m}_{Q}(\mu)\left(\sqrt{2} G_{F}\right)^{1 / 2}\left(-\epsilon_{V}^{*} \cdot \epsilon_{\gamma}^{*}+\frac{\epsilon_{V}^{*} \cdot p_{\gamma} p \cdot \epsilon_{\gamma}^{*}}{p_{\gamma} \cdot p}\right) \frac{f_{V}^{\perp}(\mu)}{f_{V}^{\perp}\left(\mu_{0}\right)} f_{V}^{\|} \frac{m_{V}}{2 \bar{m}_{Q}} \\
& \times\left[1-\frac{2}{3}\left\langle v^{2}\right\rangle_{V}+\frac{\alpha_{s}\left(\mu_{0}\right) C_{F}}{4 \pi}\left(-\log \frac{\mu_{0}^{2}}{\bar{m}_{Q}^{2}}-4\right)\right] \\
& \times\left[\mathcal{M}^{(0,0)}(\mu)+\frac{\alpha_{s}(\mu)}{4 \pi} \mathcal{M}^{(1,0)}(\mu)+\frac{\alpha_{s}\left(\mu_{0}\right)}{4 \pi} \mathcal{M}^{(0,1)}(\mu)+\left\langle v^{2}\right\rangle_{V} \mathcal{M}^{\left(0, v^{2}\right)}(\mu)\right] .
\end{aligned}
$$

\footnotetext{
${ }^{1}$ The calculations in Refs. [5,6] also included a class of corrections of order $v^{4}$ and higher. However, there are additional corrections of order $v^{4}$ that were not considered in those papers. In order to streamline the discussion, we show explicitly only contributions through order $v^{2}$ in the present paper.
} 
Here, $e(>0)$ is the electric charge at momentum scale zero, $\kappa_{Q}$ is a factor that accounts for deviations of the $H Q \bar{Q}$ coupling from the standard model $(\mathrm{SM})$ value $\left(\kappa_{Q}=1\right.$ is the SM value), $G_{F}$ is the Fermi constant, $p$ and $\epsilon_{V}^{*}$ are the quarkonium momentum and polarization, respectively, and $p_{\gamma}$ and $\epsilon_{\gamma}^{*}$ are the photon momentum and polarization, respectively. Each of the quantities $\mathcal{M}^{(i, j)}$ is a convolution of a component of the light-cone hard-scattering kernel at the scale $\mu$ with a component of the LCDA of the quarkonium, evolved from the scale $\mu_{0}$ to the scale $\mu$. The detailed definitions of the $\mathcal{M}^{(i, j)}$ are given in Ref. [1]. As in that work, we carry out the evolution of the LCDAs at next-to-leading-logarithmic accuracy, using the Abel-Padé method that is presented in that paper to sum the resulting series expansions in the eigenfunctions (Gegenbauer polynomials) of the leading-order evolution operator. Our uncertainties are also computed as outlined in Ref. [1].

Note that, because we include the NRQCD expansion for $f_{V}^{\perp} / f_{V}^{\|}$as an overall factor in the direct amplitude in Eq. (9), this expression contains cross terms of orders $\alpha_{s}^{2}$ and $\alpha_{s} v^{2}$ that are beyond the accuracy of the present calculation. We structure the amplitude in this way because of the possibility that the perturbation expansion of $f_{V}^{\perp} / f_{V}^{\|}$ may be better behaved in higher orders in $\alpha_{s}$ than the perturbation expansion of $f_{V}^{\|}[10-12]$. We use Eq. (9) to compute the direct amplitude, taking $\mu=m_{H}$, as in Ref. [1], but taking $\mu_{0}=\bar{m}_{Q}$, rather than $\mu_{0}=m_{Q}$ because, as we have already remarked, the pole mass is ill defined.

As was pointed out in Ref. [13], there is also a contribution to the decay process from an indirect amplitude, which arises from the decay of the Higgs boson through a $W$-boson loop or a quark loop into a photon plus a virtual photon, with the virtual photon decaying into a quarkonium. We compute the indirect amplitude as in Ref. [1].

We obtain the results that are shown in Table I for the direct and indirect amplitudes. The results in Table I for the direct amplitude are shifted by about 5\% for $H \rightarrow J / \psi+\gamma$ and by about $2 \%$ for $H \rightarrow \Upsilon(n S)+\gamma$, relative to the results that were given in Ref. [1]. The correction factor in Eq. (6) would shift the direct amplitude by about $13 \%$ for $H \rightarrow J / \psi+\gamma$ and by about 4-5\% for $H \rightarrow \Upsilon(n S)+\gamma$. Apparently, the additional refinements that we have introduced in this calculation work to reduce those shifts in the direct amplitude.

Our results for the decay rates and branching fractions are shown in Table II. The results in Table II for the rates are shifted by about $-1 \%$ for $H \rightarrow J / \psi+\gamma$, by about $-28 \%$ for $H \rightarrow \Upsilon(1 S)+\gamma$, by about $-42 \%$ for $H \rightarrow \Upsilon(2 S)+\gamma$, and by about $-49 \%$ for $H \rightarrow \Upsilon(3 S)+\gamma$, relative to the results that were given in Ref. [1]. The strong sensitivity of the rates for $H \rightarrow \Upsilon(n S)+\gamma$ to small changes in the direct
TABLE I. Values of the parameters $\alpha_{V}$ and $\beta_{V}$ in $\Gamma(H \rightarrow$ $V+\gamma)=\left|\alpha_{V}-\beta_{V} \kappa_{Q}\right|^{2} \times 10^{-10} \mathrm{GeV}$ for $V=J / \psi$ and $\Upsilon(n S)$.

\begin{tabular}{lcc}
\hline \hline$V$ & $\alpha_{V}$ & $\beta_{V}$ \\
\hline$J / \psi$ & $11.71 \pm 0.17$ & $\left(0.659_{-0.085}^{+0.085}\right)+\left(0.073_{-0.035}^{+0.035}\right) i$ \\
$\Upsilon(1 S)$ & $3.283 \pm 0.036$ & $\left(2.962_{-0.078}^{+0.078}\right)+\left(0.332_{-0.079}^{+0.079}\right) i$ \\
$\Upsilon(2 S)$ & $2.155 \pm 0.028$ & $\left(2.072_{-0.056}^{+0.056}\right)+\left(0.227_{-0.054}^{+0.054}\right) i$ \\
$\Upsilon(3 S)$ & $1.803 \pm 0.024$ & $\left(1.782_{-0.050}^{+0.050}\right)+\left(0.192_{-0.046}^{+0.046}\right) i$ \\
\hline \hline
\end{tabular}

TABLE II. SM values of $\Gamma(H \rightarrow V+\gamma)$ in units of $\mathrm{GeV}$ and $\operatorname{Br}(H \rightarrow V+\gamma)$ for $V=J / \psi$ and $\Upsilon(n S)$.

\begin{tabular}{lll}
\hline \hline$V$ & $\Gamma(H \rightarrow V+\gamma)(\mathrm{GeV})$ & $\operatorname{Br}(H \rightarrow V+\gamma)$ \\
\hline$J / \psi$ & $1.221_{-0.041}^{+0.042} \times 10^{-8}$ & $2.99_{-0.15}^{+0.16} \times 10^{-6}$ \\
$\Upsilon(1 S)$ & $2.13_{-0.69}^{+0.82} \times 10^{-11}$ & $5.22_{-1.70}^{+2.02} \times 10^{-9}$ \\
$\Upsilon(2 S)$ & $5.82_{-2.34}^{+2.93} \times 10^{-12}$ & $1.42_{-0.57}^{+0.72} \times 10^{-9}$ \\
$\Upsilon(3 S)$ & $3.72_{-1.55}^{+1.97} \times 10^{-12}$ & $0.91_{-0.38}^{+0.48} \times 10^{-9}$ \\
\hline \hline
\end{tabular}

amplitude occurs because of the near cancellation of the direct and indirect amplitudes in these processes.

Given the corrections and refinements to the calculation of the rates for $H \rightarrow V+\gamma$ that we have outlined above, we believe that the numerical results that we have presented in this paper are more accurate and reliable than those in Ref. [1] and should be used in preference to the results in Ref. [1] in future comparisons with experimental measurements.

\section{ACKNOWLEDGMENTS}

The work of G.T.B. is supported by the U.S. Department of Energy, Division of High Energy Physics, under Contract No. DE-AC02-06CH11357. The work of H. S. C. at CERN is supported by the Korean Research Foundation (KRF) through the CERN-Korea fellowship program. The work of J.-H. E. and J. L. was supported by the National Research Foundation of Korea (NRF) under Contract No. NRF-2017R1E1A1A01074699. The submitted manuscript has been created in part by UChicago Argonne, LLC, Operator of Argonne National Laboratory. Argonne, a U.S. Department of Energy Office of Science laboratory, is operated under Contract No. DE-AC0206CH11357. The U.S. Government retains for itself, and others acting on its behalf, a paid-up nonexclusive, irrevocable worldwide license in said article to reproduce, prepare derivative works, distribute copies to the public, and perform publicly and display publicly, by or on behalf of the Government. 
[1] G. T. Bodwin, H. S. Chung, J.H. Ee, and J. Lee, New approach to the resummation of logarithms in Higgs-boson decays to a vector quarkonium plus a photon, Phys. Rev. D 95, 054018 (2017).

[2] Y. Jia and D. Yang, Refactorizing NRQCD short-distance coefficients in exclusive quarkonium production, Nucl. Phys. B814, 217 (2009).

[3] G. T. Bodwin, E. Braaten, and G. P. Lepage, Rigorous QCD analysis of inclusive annihilation and production of heavy quarkonium, Phys. Rev. D 51, 1125 (1995); Erratum, Phys. Rev. D 55, 5853(E) (1997).

[4] X. P. Wang and D. Yang, The leading twist light-cone distribution amplitudes for the S-wave and $\mathrm{P}$-wave quarkonia and their applications in single quarkonium exclusive productions, J. High Energy Phys. 06 (2014) 121.

[5] G. T. Bodwin, H. S. Chung, D. Kang, J. Lee, and C. Yu, Improved determination of color-singlet nonrelativistic QCD matrix elements for $S$-wave charmonium, Phys. Rev. D 77, 094017 (2008).

[6] H. S. Chung, J. Lee, and C. Yu, NRQCD matrix elements for $S$-wave bottomonia and $\Gamma\left[\eta_{b}(n S) \rightarrow \gamma \gamma\right]$ with relativistic corrections, Phys. Lett. B 697, 48 (2011).
[7] G. T. Bodwin, H. S. Chung, J.-H. Ee, J. Lee, and F. Petriello, Relativistic corrections to Higgs boson decays to quarkonia, Phys. Rev. D 90, 113010 (2014).

[8] M. König and M. Neubert, Exclusive radiative Higgs decays as probes of light-quark Yukawa couplings, J. High Energy Phys. 08 (2015) 012.

[9] R. Tarrach, The pole mass in perturbative QCD, Nucl. Phys. B183, 384 (1981).

[10] M. Beneke, A. Signer, and V. A. Smirnov, Two-Loop Corrections to the Leptonic Decays of Quarkonium, Phys. Rev. Lett. 80, 2535 (1998).

[11] A. Czarnecki and K. Melnikov, Charmonium decays: $J / \psi \rightarrow e^{+} e^{-}$and $\eta_{c} \rightarrow \gamma \gamma$, Phys. Lett. B 519, 212 (2001).

[12] M. Beneke, Y. Kiyo, P. Marquard, A. Penin, J. Piclum, D. Seidel, and M. Steinhauser, Leptonic Decay of the $\Upsilon(1 S)$ Meson at Third Order in QCD, Phys. Rev. Lett. 112, 151801 (2014).

[13] G. T. Bodwin, F. Petriello, S. Stoynev, and M. Velasco, Higgs boson decays to quarkonia and the $H \bar{c} c$ coupling, Phys. Rev. D 88, 053003 (2013). 